\title{
Autodyne signal features of frequency-locked microwave oscillators
}

\author{
Vladyslav Ya. Noskov", Kirill A. Ignatkov, and Kirill D. Shaidurov \\ UrFU, Department of Radio Electronics and Telecommunication Systems IRIT-RTF, 620002 \\ Yekaterinburg, ul. Mira Street, 19, Russia
}

\begin{abstract}
The research results on the autodyne signal formation in microwave oscillators, which are exposed under the impact of the proper reflected emission and, at the same time, of the external frequency-locked signal are presented. The basic relations are obtained for signal analysis under the condition of the quasistatic target movements. The theoretical research results are confirmed by experimental data obtained on the example of an oscillator made on the basis of the $8 \mathrm{~mm}$-range Gunn diode.
\end{abstract}

\section{Introduction}

Autodyne transceivers (or just autodynes - AD) are widely used in various Short Range Radar Systems, in process parameter sensors and in the measuring systems in industry and in the transport as well as for various scientific applications, military systems and medicine devices [1-5]. The correct signal processing, which are received by AD, makes it possible to determine the electrophysical and kinematic characteristics of the radar objects. The structural simplicity and the low cost of microwave modules as well as their high sensitivity facilitate to the AD widespread application.

In order to search for new AD features and to improve its parameters, a number of publications were prepared concerning the research results of AD synchronised from the additional (external) microwave oscillator [5]. However, in the literature known to the authors, the features of the generation of synchronised AD (SAD) signals, which should be taken into account at its utilization, have not been sufficiently studied [6]. In this connection, the aim of this paper is to fill up this gap.

\section{The mathematical model of the synchronised autodyne}

An equivalent SAD circuit reduced to the plane of the active element (AE) is shown in Fig. 1. In this diagram, $Y_{\mathrm{OS}}$ displays the conductivity of an oscillatory system (OS). In the case of the single-circuit $\mathrm{OS}$, the expression for this conductivity (taking into account the AD load) has the following form:

$$
Y_{\mathrm{OS}} \equiv Y_{\mathrm{OS}}(\omega)=G_{\mathrm{OS}}+j B_{\mathrm{OS}}=G_{\mathrm{OS}}\left[1+j 2 Q_{\mathrm{L}}\left(\omega-\omega_{\mathrm{own}}\right) / \omega_{\mathrm{own}}\right]
$$

* Corresponding author: noskov@oko-ek.ru 
where $G_{\mathrm{OS}}=G_{\text {cav }}+G_{\mathrm{L}}$ and $B_{\mathrm{OS}}=2 G_{\mathrm{OS}} Q_{\mathrm{L}}\left(\omega-\omega_{\text {own }}\right) / \omega_{\text {own }}$ are the resistive and reactive conductivity of OS, relatively; $G_{\text {cav }}, G_{\mathrm{L}}$ are the resonator own loss conductivity and the load conductivity respectively; $Q_{\mathrm{L}}, \omega_{\text {own }}$ is the loaded Q-factor and the natural frequency of OS; $\omega$ is the current oscillator frequency. Average AE oscillation conductivity over the oscillation period with the voltage-current characteristic of the $N$-type, $Y_{\mathrm{AE}}=G_{\mathrm{AE}}+j B_{\mathrm{AE}} \equiv G_{\mathrm{AE}}(A, \omega)+j B_{\mathrm{AE}}(A, \omega)$, which in the general case is dependent on the $A$ amplitude and the current $\omega$ oscillation frequency, where $G_{\mathrm{AE}}, B_{\mathrm{AE}}$ are the resistive and reactive conductivities of $\mathrm{AE}$, respectively.

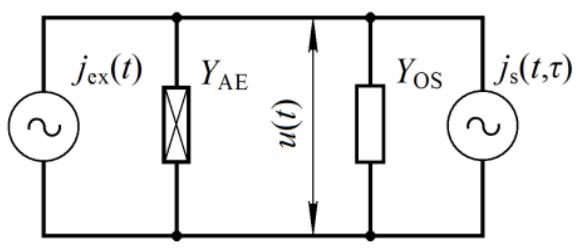

Fig. 1. The equivalent circuit diagram of SAD.

The equivalent sources of instantaneous current $j_{\mathrm{ex}}(t)$ and $j_{\mathrm{s}}(t, \tau)$ on the diagram in Fig. 1 represent the effect on $\mathrm{AD}$ of an external synchronising signal and a signal on the oscillator load caused by the reflected proper emission delayed by time $\tau$, relatively. Here $j_{\mathrm{ex}}(t)=\operatorname{Re}\left(J_{\mathrm{ex}} \exp j \omega_{\mathrm{ex}} t\right)$, where $J_{\mathrm{ex}}, \omega_{\mathrm{ex}}$ are the amplitude and the frequency of the 'external' signal. Provided that the balance of amplitudes and phases in the circuit under the influence of $\mathrm{AE}$ current, the undamped oscillations $u(t)$ will be generated. Considering usually that we have the fairly high value of the Q-factor $Q_{\mathrm{L}}$ of OS, we believe that the oscillations on $\mathrm{AE}$ are quasi-harmonic: $u(t)=\operatorname{Re}(\dot{A})=\operatorname{Re}\left[A \exp j\left(\omega_{\mathrm{ex}} t+\varphi\right)\right]$, where $A=A(t), \varphi=\varphi(t)$ are amplitude and phase, which are slowly-changing over a period of oscillations. Then, the oscillations of the equivalent source $j_{\mathrm{s}}(t, \tau)$ are also quasi-harmonic: $j_{\mathrm{s}}(t, \tau)=\operatorname{Re}\left\{J_{\mathrm{s}} \exp j\left[\omega_{\mathrm{ex}} t+\varphi-\delta(t, \tau)\right]\right\}$, where $J_{\mathrm{s}}$ is the amplitude and $\delta(t, \tau)$ is the reflected signal phase incursion.

According to Kirchhoff's laws for the circuit shown in Fig. 1, we have:

$$
\operatorname{Re}\left[\dot{A} \times\left(Y_{\mathrm{AE}}+Y_{\mathrm{OS}}\right)\right]=j_{\mathrm{s}}(t, \tau)+j_{\mathrm{ex}}(t) \text {. }
$$

To obtain the abbreviated equations of the perturbed oscillator, in expression (2), after substituting (1) into it, we first replace $\omega$ by the expression $\omega_{\mathrm{ex}}+d \varphi / d t-j(1 / A)(d A / d t)$ [7]. Here, due to the slowness of $\varphi(t)$ and $A(t)$, the values $d \varphi / d t$ and $(1 / A)(d A / d t)$ are quite small compared to the frequency $\omega_{\mathrm{ex}}$. Further, after performing the averaging operation over a period of oscillations and a series of elementary transformations, we obtain abbreviated equations for the amplitude and phase in the following form:

$$
\begin{aligned}
\frac{Q_{\mathrm{L}}}{\omega_{\mathrm{own}}} \frac{1}{A} \frac{d A}{d t}+\frac{G_{\mathrm{AE}}+G_{\mathrm{OS}}}{2 G_{\mathrm{OS}}}=\Gamma \eta \cos \delta(t, \tau)+k_{\mathrm{ex}} \eta \cos \varphi, \\
\frac{Q_{\mathrm{L}}}{\omega_{0}} \frac{d \varphi}{d t}+\frac{B_{\mathrm{AE}}+B_{\mathrm{OS}}}{2 G_{\mathrm{OS}}}=-\Gamma \eta \sin \delta(t, \tau)-k_{\mathrm{ex}} \eta \sin \varphi,
\end{aligned}
$$


where $\Gamma, k_{\mathrm{ex}}=J_{\mathrm{ex}} / G_{\mathrm{OS}} A$ are the coefficients characterising the relative levels on the oscillator load received from the radar object emitted oscillations and the external synchronising effects, respectively; $\eta=Q_{\mathrm{L}} / Q_{\mathrm{ex}}, Q_{\mathrm{ex}}$ are efficiency and the external Qfactor of OS.

We further assume that the relative level of reflected emission $\Gamma<<1$. In this case, we assume that the synchronising effect is also low $k_{\mathrm{ex}}<<1$ but it can cause significant deviations of the steady-state mode of the stand-alone oscillator in comparison with the reflected emission effects, $k_{\mathrm{ex}}>>\Gamma$. Therefore, to describe the AD behaviour, we further linearized equations (3), (4) with respect to the steady-stat mode of synchronous oscillations.

The reflected emission radiation, which produces an effect on the oscillator OS, as noted above, causes changes in the amplitude $\Delta A=A-A_{\mathrm{syn}}^{0}$ and the phase $\tilde{\varphi}=\varphi-\varphi_{0}$ of the SAD oscillations relative to their steady-state synchronous values $A_{\text {syn }}^{0}$ and $\varphi_{0}$. At the same time, we assume that the variations in the amplitude $\Delta A$ and phase $\tilde{\varphi}$ in the vicinity of the steady-state mode of oscillations in absolute values are quite low: $|\Delta A|<<A_{\text {syn }}^{0},|\tilde{\varphi}|<<\pi / 2$. Then we write the system (3), (4) for small relative variations in the amplitude $a=\Delta A / A_{\mathrm{syn}}^{0}$ and frequency mismatch $\left(\omega_{\mathrm{ex}}\right.$ and $\left.\omega_{0}\right)$ as well as the absolute $\tilde{\varphi}$ phase variations as follows:

$$
\begin{aligned}
& \frac{d a}{d t}+\frac{1}{\tau_{\mathrm{a}}} a+\frac{\operatorname{tg} \varphi_{0}}{\tau_{\varphi}} \tilde{\varphi}+\rho\left(\omega_{\mathrm{ex}}-\omega_{0}\right)=\Gamma \frac{K_{\mathrm{a}}}{\tau_{\mathrm{a}}} \cos \delta(t, \tau), \\
& \frac{d \tilde{\varphi}}{d t}+\frac{\gamma}{\tau_{\mathrm{a}}} a+\frac{1}{\tau_{\varphi}} \tilde{\varphi}+\left(\omega_{\mathrm{ex}}-\omega_{0}\right)=-\Gamma \frac{K_{\mathrm{a}}}{\tau_{\mathrm{a}}} \sin \delta(t, \tau),
\end{aligned}
$$

where $K_{\mathrm{a}}=\eta / \alpha$ is the autodyne gain coefficient; $\tau_{\mathrm{a}}, \tau_{\varphi}$ are time constants of changes (relaxation) in the amplitude and phase, respectively:

$$
\tau_{\mathrm{a}}=Q_{\mathrm{L}} / \alpha \omega_{0}, \tau_{\varphi}=Q_{\mathrm{ex}} / k_{\mathrm{ex}} \omega_{0} \cos \varphi_{0}
$$

$\gamma=\beta / \alpha, \rho=\varepsilon / Q_{\mathrm{L}}$ are non-isochronous and non-isodromic coefficients of synchronised $\mathrm{AD}$ respectively; $\quad \alpha=\left(A_{0} / 2 G_{\mathrm{OS}}\right)\left(\partial G_{\mathrm{AE}} / \partial A\right)_{0}, \quad \varepsilon=\left(\omega_{0} / 2 G_{\mathrm{OS}}\right)\left[\partial\left(G_{\mathrm{AE}}+G_{\mathrm{OS}}\right) / \partial \omega\right]$, $\beta=\left(A_{0} / 2 G_{\mathrm{OS}}\right)\left(\partial B_{\mathrm{AE}} / \partial A\right)_{0}$ are parameters characterising the strength of the limit cycle, non-isodromity and non-isochronity of the oscillator, respectively;

$$
\varphi_{0}=-\left(\theta_{0}+\arcsin \chi_{\mathrm{n}}\right),
$$

$\theta_{0}=\operatorname{arctg} \gamma$ is the phase characteristic's offset angle; $\chi_{\mathrm{n}}=\left(\omega_{\mathrm{ex}}-\omega_{0}\right) / \Delta_{m}$ is the normalized mismatch of frequencies $\omega_{\mathrm{ex}}$ and $\omega_{0} ; \Delta_{m}$ is the half-width of the oscillator synchronisation band:

$$
\Delta_{m}=k_{\mathrm{ex}} \frac{\omega_{0}}{Q_{\mathrm{ex}}} \frac{\left(1+\gamma^{2}\right)^{1 / 2}}{(1-\gamma \rho)} .
$$


The resulting system of linearized equations (5) and (6), taking into account the wellknown duality principle, has sufficient generality for the SAD analysis with any type of AE.

\section{Calculation of the parameters of the autodyne signal of SAD}

To obtain analytical solutions of differential equations describing the behaviour of selfoscillating systems, the so-called quasi-static method is widely used [8]. This method makes it relatively easy to calculate the AD characteristics that determine the generation of their output signals. To find these characteristics, we set the derivatives in (5) and (6) to be zero and take into account that in the synchronous mode $\omega=\omega_{\mathrm{ex}}$ there is also a phase incursion $\delta(t, \tau)=\omega_{\mathrm{ex}} \tau$. Then from the resulting system of algebraic equations, we obtain expressions for autodyne variations in the phase $\tilde{\varphi}(\tau)$ and amplitude $a(\tau)$ of SAD:

$$
\begin{aligned}
& \tilde{\varphi}(\tau)=-\left(k_{\mathrm{ex}} / Q_{\mathrm{ex}}\right) h_{\varphi} \chi-\left(\Gamma / k_{\mathrm{ex}}\right) H_{\varphi} \sin \left(\omega_{\mathrm{ex}} \tau+\theta\right), \\
& a(\tau)=-K_{\mathrm{a}} Q_{\mathrm{ex}} h_{\mathrm{a}} \chi+\Gamma K_{\mathrm{a}} H_{\mathrm{a}} \cos \left(\omega_{\mathrm{ex}} \tau-\varphi_{0}\right),
\end{aligned}
$$

where $h_{\varphi}, h_{\mathrm{a}}, H_{\varphi}, H_{\mathrm{a}}$ are transfer coefficients due to the internal parameters of the oscillator and conditions for its synchronisation:

$$
\begin{aligned}
& h_{\varphi}=\frac{1-\gamma \rho}{\left(1-\gamma \operatorname{tg} \varphi_{0}\right) \cos \varphi_{0}}, h_{\mathrm{a}}=\frac{\rho-\operatorname{tg} \varphi_{0}}{\left(1-\gamma \operatorname{tg} \varphi_{0}\right)}, \\
& H_{\varphi}=\frac{\left(1+\gamma^{2}\right)^{1 / 2}}{\left(1-\gamma \operatorname{tg} \varphi_{0}\right) \cos \varphi_{0}}, H_{\mathrm{a}}=\frac{1}{\left(1-\gamma \operatorname{tg} \varphi_{0}\right) \cos \varphi_{0}} .
\end{aligned}
$$

As can be seen from (10), (11), the first terms of the right-hand sides determine the level of the constant component relative to the steady-state mode of the stand-alone oscillator, which is due to the action of an external synchronising signal only. The second terms in these expressions are associated with the effect of the reflected microwave emission; they determine the creation of the dependences of the instantaneous values of the variations in the oscillation amplitude and phase on the delay time of the reflected emission.

The first dependence in the AD theory is usually called the amplitude characteristic [9], the second one is called the phase characteristic of SAD. In contrast to the characteristics of ordinary (non-synchronised) AD, the SAD characteristics, as can be seen from (10), (11), are harmonic functions of the delay time $\tau$ of the reflected emission. Fig. 2 presents dependency diagrams of the coefficients $h_{\varphi}\left(\chi_{\mathrm{n}}\right), h_{\mathrm{a}}\left(\chi_{\mathrm{n}}\right), H_{\varphi}\left(\chi_{\mathrm{n}}\right)$ and $H_{\mathrm{a}}\left(\chi_{\mathrm{n}}\right)$ calculated according to (12), (13) taking into account (9) at various values of the non-isochronous coefficient $\gamma$. From diagrams Fig. 2, $a$, it can be seen that the function $h_{\varphi}\left(\chi_{\mathrm{n}}\right)$ has central symmetry with respect to the origin of coordinates. Moreover, variations in the coefficients $\gamma$ and $\rho$, as shown by the calculations, cause only changes in the angle of slope of curves. The function $h_{\mathrm{a}}\left(\chi_{\mathrm{n}}\right)$, in the case of an isochronous oscillator, when $\gamma=0$, has axial symmetry (see curve 1 in Fig. 2, b). Moreover, variations in the coefficients $\gamma$ and $\rho$ cause disturbances in this symmetry while maintaining a significant increase in the coefficients $h_{\varphi}\left(\chi_{\mathrm{n}}\right)$ и $h_{\mathrm{a}}\left(\chi_{\mathrm{n}}\right)$ at the edges of the synchronisation band (see curves 2 and 3 in Fig. 2, b). 


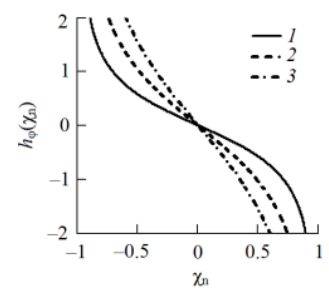

(a)

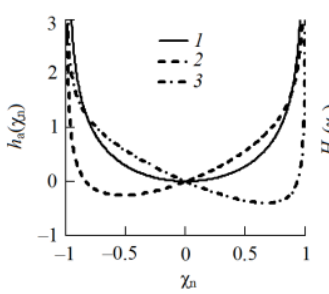

(b)

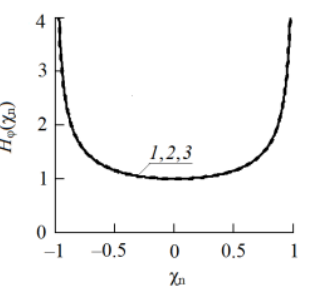

(c)

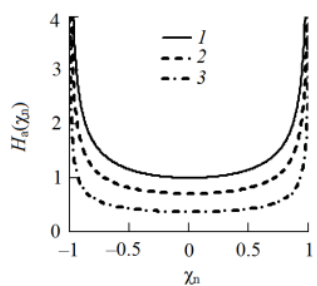

(d)

Fig. 2. Curves $h_{\varphi}\left(\chi_{\mathrm{n}}\right)(a), h_{\mathrm{a}}\left(\chi_{\mathrm{n}}\right)(b), H_{\varphi}\left(\chi_{\mathrm{n}}\right)(c)$ and $H_{\mathrm{a}}\left(\chi_{\mathrm{n}}\right)(d)$ calculated at various values of the coefficient $\gamma: \gamma=1.5$ (curves 1 ), $\gamma=0$ (curves 2), $\gamma=-2.5$ (curves 3).

The curves $H_{\varphi}\left(\chi_{\mathrm{n}}\right)$ and $H_{\mathrm{a}}\left(\chi_{\mathrm{n}}\right)$ (see curves Fig. 2, c, d), have the axial symmetry over the ordinate axis for all coefficient variations $\gamma$. In the centre of the synchronisation band, the transfer coefficients have the lowest values, and toward the edges of the synchronisation band, their rapid growth is recorded. In this case, the value of the transfer coefficient $H_{\mathrm{a}}\left(\chi_{\mathrm{n}}\right)$ in the centre of the isochronous $(\gamma=0)$ SAD synchronisation band, is equal to 1 (see curve 1 in Fig. 2, d). Therefore, in this detuning region $\chi_{\mathrm{n}}$, the values of the autodyne gain coefficients of SAD and ordinary AD coincide.

In the case of a non-synchronous oscillator $(\gamma \neq 0)$, the resulting SAD transfer coefficient equal to the product $K_{\mathrm{a}} H_{\mathrm{a}}$, due to the decrease in the value of $H_{\mathrm{a}}$ (see curves 2 and 3 in Fig. 2, d), has lower values than the value of the autodyne gain coefficient of the usual $\mathrm{AD} K_{\mathrm{a}}$. Moreover, if you introduce the initial detuning between the frequencies $\omega_{\mathrm{ex}}$ and $\omega_{0}$ closer to the edge of the synchronisation band, then in SAD there is the possibility of a significant increase in the transfer coefficient of the autodyne signal compared to conventional AD.

It should be noted that the conclusions made are valid subject to inequality $T_{\mathrm{a}} \gg \tau_{\mathrm{a}}$, where $T_{\mathrm{a}}=2 \pi / \Omega_{\mathrm{D}}$ is the autodyne signal period, $\Omega_{\mathrm{D}}$ is its frequency equalled to the Doppler frequency. This inequality is satisfied under the condition of a sufficiently 'slow' movement of the reflecting object, in which $\mathrm{AD}$ manages to 'respond' to changes in the phase $\delta(t, \tau)$ of the reflection coefficient. For a more accurate determination of the limiting capabilities of SAD, we further consider the dynamic characteristics of AD in the case of 'fast' movement of a location object.

\section{Experimental results}

As a target of research, we used the 8-mm hybrid-integrated oscillator module of the 'Tigel08M' type, which had been made on the basis of the Gunn planar diode. Its supply voltage is 4 $\mathrm{V}$, with a consumption current of about $0.2 \mathrm{~A}$. The module generation frequency is $37.5 \mathrm{GHz}$, the output power is $15 \mathrm{~mW}$. To register a signal by changing the oscillation amplitude, a detector diode with the Schottky barrier is built into the topology of the module [9].

Experimental research into the module was carried out on the test bench, a description of which is presented in [9]. The AD under study in the test bench is connected to the Doppler signal simulator (DSS) by the waveguide path, which also contains a variable attenuator simulating the attenuation of the radiation propagating in space, and a directional coupler. One of the arms of the coupler is connected to a measuring oscillator, the output signal of which is used to synchronise AD. The power of the synchronisation signal in all experiments was maintained equal to $1 \mathrm{~mW}$, while the half-width of the synchronisation band was $\Delta_{m}=2 \pi \times 50 \times 10^{6}$. 
The length of the waveguide between $\mathrm{AD}$ and the simulator, which simulates the delay of the reflected radiation in the test bench, had a length $12 \mathrm{~m}\left(\tau \approx 100 \times 10^{-9} \mathrm{~s}\right)$. A variable attenuator set the attenuation in the path of $20 \mathrm{~dB}$, the autodyne frequency deviation was $\Delta \omega_{m} \approx 2 \pi \times 1.3 \times 10^{6}$. In this case, the calculated value of the return connection parameter was provided $C_{\mathrm{FB}}=\Delta \omega_{m} \tau \approx 0.8$ [14]. Usually at this value $C_{\mathrm{FB}} \mathrm{SAD}$ signals have a noticeable level of anharmonic distortion, which is confirmed by the waveform of the signal $u_{\mathrm{s}}(t)$ presented in Fig. 3, $a$ from the output of the diode detector.

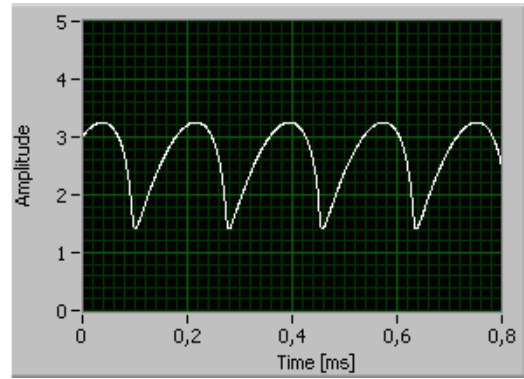

(a)

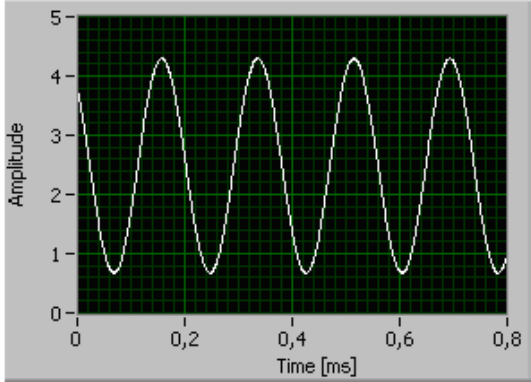

(c)

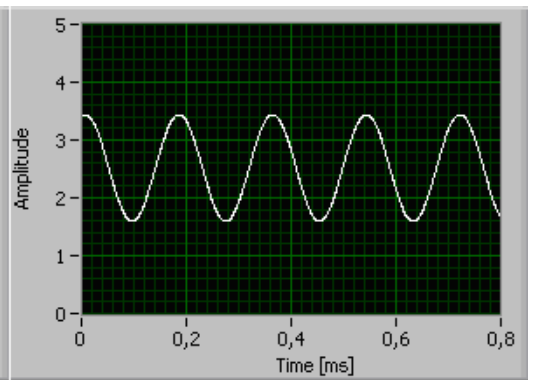

(b)

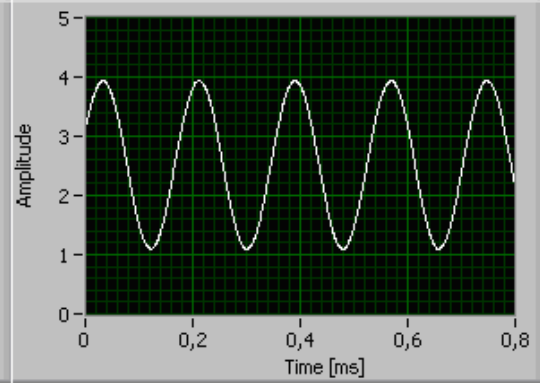

(d)

Fig. 3. Oscillograms of SAD signals $u_{\mathrm{s}}(t) \mathrm{SAD}$ on the Gunn diode obtained in the absence of synchronisation $(a)$; and if available for cases where $\chi_{\mathrm{n}}=0(b), \chi_{\mathrm{n}}=-0.8(c)$ and $\chi_{\mathrm{n}}=0.8(d)$.

The remaining oscillograms in Fig. $3(b-d)$ are obtained when a synchronising signal is applied to $\mathrm{AD}$ for three cases, the frequency $\omega_{\mathrm{ex}}$ corresponds to the middle of the synchronisation band $\omega_{0}(b)$, the presence of the initial detuning frequency $\omega_{\mathrm{ex}}$ down $(c)$ and up $(d)$ relative to the frequency $\omega_{0}$ by $0.8 \Delta_{m}$. The speed of movement of the DSS reflector in all cases was kept constant and was $20 \mathrm{~m} / \mathrm{s}$.

From a comparison of the oscillograms of Fig. 3 it can be seen that the SAD signals $u_{\mathrm{s}}(t)$ are virtually harmonic. It should be noted that in the middle of the synchronisation band, the amplitude of the autodyne signal is the lowest (see Fig. 3, b), and in approaching its edges, it increases significantly (see Fig. 3, c, d). The results obtained qualitatively confirm the course of the curves on Fig. 2, $d$.

\section{Conclusion}

Thus, a mathematical model of an autodyne transceiver stabilized in frequency by a signal from an additional oscillator has been developed. The basic relationships for calculating its signal characteristics are obtained. It is found that the AD synchronisation from an external oscillator eliminates the anharmonic distortion of signals typical of ordinary (non- 
stabilized) $\mathrm{AD}$, which contributes to the expansion of their dynamic range. It is demonstrated that in the synchronized $\mathrm{AD}$ by introducing the initial detuning between the frequencies of the external oscillator and the autodyne frequency within the synchronisation band, there is the possibility of the significant increase in the transfer coefficient of the autodyne signal compared to conventional AD.

\section{References}

1. D.A. Usanov, Al.V. Skripal, An.V. Skripal, A.E. Postelga. Instrum. \& Experimental Techn. 47, 5. (2004)

2. S.A. Alidoost, R. Sadeghzade, R. Fatemi. 11th Intern. Radar Symp. (IRS-2010). Vilnius, Lithuania. 2, 406-409 (2010)

3. D.A. Usanov, A.E. Postelga. Biomedical Engineering. 45, 1. (2011)

4. G.P. Ermak, I.V. Popov, A.S. Vasiliev, A.V. Varavin, V.Ya. Noskov, K.A. Ignatkov. Telecommunication \& Radio Engineering. 71, 6. (2012)

5. I.V. Komarov, S.M. Smolskiy. Fundamentals of Short-Range FM Radar. Artech House, Norwood, MA, USA (2003)

6. V.Ya. Noskov, K.A. Ignatkov. Russian Physics Journal. 56, 12. (2013)

7. K. Kurokava. Proceedings of the IEEE. 61, 10. (1973)

8. V.Ya. Noskov, K.A. Ignatkov. Radioelectron. \& Communicat. Systems. 57, 3. (2014)

9. V.Ya. Noskov, K.A. Ignatkov. Journal Communic. Technol. \& Electron. 61, 9. (2016) 